\title{
Reward Anticipation Is Differentially Modulated by Varenicline and Nicotine in Smokers
}

\author{
John R Fedota*,, Matthew T Sutherland ${ }^{2}$, Betty Jo Salmeron', Thomas J Ross', L Elliot Hong ${ }^{3}$ and \\ Elliot A Stein' \\ 'Neuroimaging Research Branch, National Institute on Drug Abuse, Intramural Research Program, National Institutes of Health, Baltimore, MD, \\ USA; ${ }^{2}$ Department of Psychology, Florida International University, Miami, FL, USA; ${ }^{3}$ Maryland Psychiatric Research Center, University of Maryland \\ School of Medicine, Baltimore, MD, USA
}

Recidivism rates for cigarette smokers following treatment often exceed $80 \%$. Varenicline is the most efficacious pharmacotherapy currently available with cessation rates of 25-35\% following a year of treatment. Although the in vivo binding properties are well known, varenicline's neurobiological mechanisms of action are still poorly understood. Varenicline acts as a nicotinic receptor partial agonist or antagonist depending on the presence or absence of nicotine and has been implicated in the reduction of reward signaling more broadly. The current study probed anticipatory reward processing using a revised monetary incentive delay task during fMRl in cohorts of smokers and non-smokers who completed a two-drug, placebo-controlled, double-blind crossover study. All participants underwent $~ 17$ days of order-balanced varenicline and placebo pill administration and were scanned under each condition wearing a transdermal nicotine or placebo patch. Consistent with nicotine's ability to enhance the rewarding properties of nondrug stimuli, acute nicotine administration enhanced activation in response to reward-predicting monetary cues in both smokers and non-smokers. In contrast, varenicline reduced gain magnitude processing, but did so only in smokers. These results suggest that varenicline's downregulation of anticipatory reward processing in smokers, in addition to its previously demonstrated reduction in the negative affect associated with withdrawal, independently and additively alter distinct brain circuits. These effects likely contribute to varenicline's efficacy as a pharmacotherapy for smoking cessation. Neuropsychopharmacology (20I5) 40, 2038-2046; doi: I 0. I038/npp.20I5.54; published online 25 March 2015

\section{INTRODUCTION}

Current neurobiological models of drug addiction emphasize the importance of mesocorticolimbic (MCL) pathways in processing the reinforcing aspects of abused drugs during the initiation and maintenance of addiction (Everitt and Robbins, 2005; Goldstein and Volkow, 2011; Koob and Volkow, 2009). The MCL consists predominantly of dopaminergic projections from the midbrain ventral tegmental area to limbic and cortical projection fields in nucleus accumbens (NAc), amygdala, orbitofrontal cortex (OFC), and anterior cingulate cortex (ACC). In the case of tobacco smoking, reinforcing and subsequent addictive effects of nicotine are the result of neuronal acetylcholine receptor (nAChR) activation that modulates 'downstream' events including increased MCL dopamine (DA) release (Tapper et al, 2004; De Biasi and Dani, 2011). The addictive propensity of nicotine is theorized to be due to its quick but short-lived agonistic effect on nAChRs containing $\alpha 4$ and $\beta 2$ subunits located on the

*Correspondence: Dr JR Fedota, Neuroimaging Research Branch, National Institute on Drug Abuse, Intramural Research Program, National Institutes of Health, NIDA-IRP, 25I Bayview Boulevard, Baltimore, MD 21224, USA, Tel: 443740 2782, Fax: 443740 2753, E-mail: john.fedota@nih.gov

Received I4 November 2014; revised 9 February 2015; accepted 10 February 2015; accepted article preview online 6 March 2015 presynaptic membrane of DA neurons (Exley et al, 2011; Tapper et al, 2004).

Varenicline (VAR) is a partial agonist/antagonist at the $\alpha 4 \beta 2 \mathrm{nAChR}$ (Rollema et al, 2009), and an effective clinical aid in smoking cessation (Oncken et al, 2006). Preclinical evidence suggests that VAR stimulates DA release along the MCL pathway, but to a smaller extent and over a longer duration than nicotine (Coe et al, 2005; Rollema et al, 2009). When administered alone, VAR acts as a partial agonist, with effects $\sim 50-60 \%$ of nicotine. However, when VAR and nicotine are administered in concert (as prescribed clinically with individuals initially allowed to continue smoking as usual), VAR's higher binding affinity antagonizes nicotine leading to reduced activation when compared with nicotine administration alone. This partial agonist/antagonist pharmacokinetic profile has recently been demonstrated in vivo within the amygdala and limbic circuitry using fMRI (Sutherland et al, 2013a,b).

Although not previously examined, similar predictions of partial agonism/antagonism can be made about VAR within the reward circuitry. VAR has been broadly implicated in the reduction of reward signaling, selectively decreasing voluntary alcohol intake in both rodents (Hendrickson et al, 2010; Steensland et al, 2007) and humans (Fucito et al, 2011; McKee et al, 2009). Administration of VAR decreases subjective reports of nicotine craving (Brandon et al, 2011; 
Patterson et al, 2009) and smoking cue-induced activity in the medial OFC (Franklin et al, 2011) in nontreatmentseeking smokers. In addition, VAR reduces subjective reward of nicotine during lapses in smoking cessation (Oncken et al, 2006; Patterson et al, 2009).

Recent evidence (Rose et al, 2013) using a revised monetary incentive delay (MID) task (Knutson et al, 2000, 2001) showed that chronic smoking was associated with reduced valence-dependent activity in the MCL DA reward pathway, whereas acute nicotine administration enhanced striatal activation to stimuli, indicating the magnitude of impending gains (Rose et al, 2013). These findings suggest that the interaction between chronic and acute nicotine exposure has dissociable effects on reward anticipation, which can be localized to the MCL DA reward pathway.

On the basis of these data and employing the previously described fully crossed nicotine/VAR administration design (Sutherland et al, 2013a,b), we compared the effects of nicotine and VAR on anticipatory reward processing. We hypothesized that (a) when administered alone the partial agonist/antagonist profile of VAR would result in a blunted but anatomically similar activation profile in the MCL DA pathway as that seen with nicotine administration and (b) when administered in combination with nicotine, VAR would partially antagonize nicotine's agonist properties on MCL reward processing.

\section{MATERIALS AND METHODS}

\section{Participants}

Two groups of healthy participants (24 smokers and 20 nonsmoking controls) all of whom were right-handed, 18-55 years of age, and matched for age and gender (Table 1) completed the study. Exclusionary criteria included a history of drug dependence (aside from nicotine in smokers), neurological or psychiatric disorders, cardiovascular or renal impairment, or diabetes. Only daily, non-treatment-seeking smokers reporting smoking $\geqslant 10$ cigarettes per day for $\geqslant 2$ years were included. All participants completed six MRI days over the $\sim 6$-week course of the study. Data from one smoker and two non-smokers were excluded from neuroimaging analyses because of excessive head motion during one or more scanning sessions. Before entering the study, all

Table I Participant Demographics

\begin{tabular}{lccc}
\hline & $\begin{array}{c}\text { Smoker } \\
(\boldsymbol{n}=\mathbf{2 3} \text {; mean; SE) }\end{array}$ & $\begin{array}{c}\text { Non-smoker } \\
(\boldsymbol{n}=\mathbf{~ I 8 ; ~ m e a n ; ~ S E ) ~}\end{array}$ & p-value \\
\hline Age & $36(\mid 0)$ & $3 \mid(7)$ & 0.09 \\
Education (years) & $\mid 3.70(\mid .89)$ & $\mid 5.06(1.30)$ & $0.0 \mid$ \\
IQ & $\mid 08(\mid 2)$ & $\mid 14(\mid 3)$ & 0.04 \\
Gender (M/F) & $|2 /| \mid$ & $9 / 9$ & \\
CPD & $|6.4|(7.70)$ & - & \\
Years smoking & $\mid 7.69(\mid 0.69)$ & - & \\
FTND & $4.87(\mid .8)$ & - & \\
Age of first cig & $\mid 5.78(3.7 \mid)$ & - \\
\hline
\end{tabular}

Abbreviations: CPD, cigarettes per day; FTND, Fagerström test of nicotine dependence. participants gave written informed consent in accordance with the Institutional Review Board of the National Institute on Drug Abuse Intramural Research Program (NIDA-IRP). Volunteers were remunerated for their participation.

\section{Experimental Design}

As previously described (Sutherland et al, 2013a), the experiment consisted of a two-drug, placebo-controlled, double-blind crossover design. Participation involved a total of nine study visits (one orientation, two neurocognitive assessments, two baseline imaging scan days (nicotine and placebo patch conditions in the absence of any pill, data not reported here), and four drug (pill) plus patch administration imaging scan days; Supplementary Fig S1). Following the baseline scans, all participants were administered counterbalanced courses of VAR $(17.0 \pm 4.2$ days $)$ and placebo pill $(16.5 \pm 3.4$ days) and were scanned under each condition wearing a transdermal nicotine or placebo patch at the end of each drug administration arm.

Varenicline (Chantix, Pfizer, New York, NY) and placebo pills were distributed in identical blister packs according to the standard dosage guidelines (www.pfizer.com/products). Medication adherence and side effects were monitored through regular telephone assessments and at in-person visits. Percent of pills that participants removed from blister packs out of the number of pills that were to have been consumed was calculated. Across all participants, average adherence was $96.5 \pm 0.7 \%$ over the two $\sim 17$-day medication periods (varenicline: $96.5 \pm 0.9 \%$; placebo: $96.5 \pm 0.7 \%$ ). Adherence was lower for smokers $(95.1 \pm 1.2 \%)$ in comparison with non-smokers $(98.2 \pm 0.4 \%$; GROUP effect: $F$ $[1,42]=5.2, p=0.03)$ in the absence of a GROUP $\times$ PILL interaction $(p>0.8)$. Participants confirmed taking a study pill upon arrival for each scan day. Transdermal nicotine (NicoDerm CQ, GlaxoSmithKline, Research Triangle Park, NC) or placebo patches were applied to the upper back at the beginning of each neuroimaging visit. Non-smokers were administered $7 \mathrm{mg}$ nicotine patches. For smokers, a variable patch dosing strategy to match daily nicotine intake was used: $21 \mathrm{mg}$ (10-15 cigs/day; $n=10), 28 \mathrm{mg}$ (16-20 cigs/day; $n=9), 35 \mathrm{mg}(21-25 \mathrm{cigs} /$ day; $n=1)$, and $42 \mathrm{mg}$ ( $>25 \mathrm{cigs} /$ day; $n=3)$. Patches were worn for the duration of each neuroimaging visit $(\sim 9 \mathrm{~h})$.

\section{Procedures}

Smokers were instructed to have their last cigarette $12 \mathrm{~h}$ before the scheduled arrival for each imaging scan. Upon arrival, participants were tested for recent illicit drug (methadone, benzodiazepines, cocaine, amphetamine/ methamphetamine, opiates, barbiturates, tetrahydrocannabinol, and tricyclic antidepressants using Triage urine assay) and alcohol use (Breathalyzer), and for expired carbon monoxide (CO) levels. For smokers, $\mathrm{CO}$ levels were lower on scan visits $(6.9 \pm 2.6$ p.p.m.) than baseline visits $(18.6 \pm 8.9$ p. p.m.; $p<0.001)$; non-smokers' CO levels did not differ across visits $(1.9 \pm 0.3$ vs $1.8 \pm 0.4$ p.p.m.; $p=0.3)$.

During each imaging scan visit, participants completed two MRI scanning sessions, each lasting $\sim 2 \mathrm{~h}$ and separated by $\sim 2 \mathrm{~h}$ (Supplementary Table 1 ). The revised MID task was 
completed during the afternoon session $\sim 6-7 \mathrm{~h}$ after nicotine or placebo patch administration.

\section{Revised MID Task}

Participants completed a revised version of the MID task (MID-R; (Rose et al, 2013)) designed to ascertain the brain circuitry involved in the anticipation and receipt of monetary losses and gains (Knutson et al, 2000, 2001).

For participants, the task goal was to maximize overall winnings by minimizing losses and maximizing gains. Participants made a speeded response before a visual target stimulus presentation ended. Initially, the visual target was presented for $250 \mathrm{~ms}$. The duration of target presentation was increased or decreased on the basis of the speed of response in a staircase procedure in 25-ms increments in an attempt to ensure that participants were successful in $~ 66 \%$ of task trials. Following target presentation, feedback was presented illustrating the success or failure and the magnitude of money gained or lost on the current trial as well as the running total of compensation. Participants completed four 8-min task blocks in the MRI scanner, consisting of a total of 85 gain, 85 loss, and 28 neutral trials across the experiment. Sixty-four rest (null) trials were included to add temporal jitter.

The principal difference in the MID-R task is the temporal separation of cues of trial valence (positive, negative, and neutral) and trial magnitude (high, medium, low, and neutral); the original task combined both valence and magnitude into a single cue presentation. On the basis of prospect theory (Kahneman and Tversky, 1979), the magnitude of gains and losses was asymmetrically manipulated. High, medium, and low GAIN trial magnitudes were equal to $\$ 15, \$ 10$, and $\$ 2.50$, while high, medium, and low LOSS trial magnitudes were $-\$ 9,-\$ 6$, and $-\$ 1.50$, respectively. For detailed task description, refer to Supplementary Fig S2 and Rose et al (2013).

\section{Functional MRI}

Whole-brain echo planar images were acquired on a 3T Siemens Allegra scanner (Erlangen, Germany). Oblique axial $\left(39.4 \mathrm{~mm} ; 30^{\circ}\right.$ to anterior commissure-posterior commissure) slices were acquired using a T2* weighted, single-shot gradient echo, echo planar imaging sequence sensitive to blood oxygenation level-dependent (BOLD) effects (234 volumes; repetition time $(\mathrm{TR})=2000 \mathrm{~ms}$; echo time $(\mathrm{TE})=$ $27 \mathrm{~ms}$; flip angle $(\mathrm{FA})=80 \mathrm{deg}$; field of view $=220 \times 220 \mathrm{~mm}$; image matrix $=64 \times 64)$. High-resolution oblique-axial structural images were also acquired using a 3D magnetization prepared rapid gradient-echo (MPRAGE) T1-weighted sequence $(\mathrm{TR}=2500 \mathrm{~ms} ; \mathrm{TE}=4.38 \mathrm{~ms} ; \mathrm{FA}=8 \mathrm{deg}$; voxel size $=1 \mathrm{~mm}^{3}$ ).

\section{Data Analysis}

Reaction time (RT) data were analyzed on the basis of a GROUP (smoker/non-smoker) $\times$ PILL (VAR/placebo $) \times$ PATCH (nicotine/placebo) mixed effects ANOVA model using $R$ Project for Statistical Computing (http://www. $\mathrm{R}$-project.org). Imaging data were analyzed using Analysis of Functional NeuroImages (AFNI; Cox, 1996). Functional data were slice-time and motion-corrected, and aligned with anatomical images. Following motion correction, motion censoring was performed on any two consecutive time points with derivative values greater than $0.3 \mathrm{~mm}$. Time series were normalized to percent signal change and spatially smoothed to an 8-mm full-width at half-maximum (Friedman et al, 2006). The data were submitted to a voxel-wise multiple regression with regressors expressed as a delta function convolved with a standard hemodynamic response function and its temporal derivative. Regressors included trial valence cue (gain, loss, and neutral), trial magnitude cue (high, medium, low, and zero), and trial feedback (correct and error) as well as six head motion parameters. A voxel-wise average amplitude change equal to the percentage change from baseline $(\beta)$ was calculated per participant, regressor, and session. While both positive and negative cues for valence and magnitude were analyzed, these cues were not directly compared with one another, only to neutral cues. This procedure was on the basis of previous evidence showing larger increases in activation within the MCL DA pathway in response to anticipatory processing of gains as compared with losses (Knutson et al, 2001; O'Doherty et al, 2002).

Group-level imaging analysis was performed separately on anticipatory cues of valence (gain-neutral and loss-neutral), gain magnitude (high, medium, and low), and loss magnitude (high, medium, and low) using multivariate modeling in AFNI via 3dMVM (Chen et al, 2013). For cues of trial valence, data were analyzed in separate GROUP (smoker/ control $) \times$ PILL $($ VAR/placebo $) \times$ PATCH (nicotine/placebo) mixed effects ANOVAs individually for positive and negative valence cues. Likewise, for cues of trial magnitude, data were analyzed in separate GROUP (smoker/non-smoker) $\times$ MAGNITUDE (high/medium/low) $\times$ PILL $($ VAR/Placebo $) \times$ PATCH (nicotine/placebo) mixed effects ANOVAs individually for gain and loss magnitude cues.

Given VAR's $\sim 24 \mathrm{~h}$ half-life (Faessel et al, 2006), we assumed that carryover effects were negligible in those participants first receiving active medication as subsequent scanning under placebo pills occurred $\sim 2$ weeks after the last active dose. Further, when pill ORDER (VAR-first $v s$ placebo-first) was included in the statistical model (that is, ORDER $\times$ GROUP $\times$ MAGNITUDE $\times$ PILL $\times$ PATCH $)$ no significant ORDER-related effects or interactions were observed nor were any of the results discussed below altered by the inclusion of ORDER in the model.

Consistent with our previous study (Rose et al, 2013), region of interest (ROI) analysis was performed in a priori hypothesized and small volume-corrected (SVC) rewardpathway regions (Supplementary Fig S3). Bilateral ROIs were placed in the striatum (NAcc, caudate, and putamen) and medial prefrontal cortex (Brodmann's area (BA) 10, corresponding to the superior frontal gyrus (SFG) and BA 32 , corresponding to the ACC). Striatal regions were defined using a probabilistic atlas (DD_Desai_MPM) in AFNI, which provided the best anatomical overlap with striatal structures. Cortical regions were defined using a Talairach template.

Voxel-wise thresholds corrected for multiple comparisons were calculated using Monte Carlo simulations. Significance was determined as meeting or exceeding minimum cluster extent criteria at $p_{\text {corrected }} \leq 0.05$. This correction accounted for the total ROI/SVC volume. The direction of significant results was confirmed with corrected $(p<0.05)$ contrasts. 


\section{RESULTS}

\section{Behavioral Results}

Across both smokers and non-smokers, the hypothesized PILL $\times$ PATCH interaction was observed $(\mathrm{F}(1,39)=7.38$, $p=0.01$; Supplementary Fig S4) such that nicotine (vs placebo patch) was associated with reduced RT when administered with placebo pill (RT nicotine $=297 \mathrm{~ms}$, RT placebo patch $=307 \mathrm{~ms} ; \mathrm{F}(1,39)=10.99, p<0.005)$, an effect that was absent with VAR administration $(\mathrm{RT}$ nicotine $=304$, RT placebo patch $=300 ; \mathrm{F}(1,39)=1.10, p>0.05)$. In contrast, VAR ( $v s$ placebo pill) was associated with reduced RT when administered with placebo patch (RT VAR $=300$, RT placebo pill $=307 ; \mathrm{F}(1,39)=4.37, p<0.05)$, an effect that was reversed with nicotine administration $(\mathrm{RT} \mathrm{VAR}=304$, RT placebo pill $=297, \mathrm{~F}(1,39)=6.16, p<0.05)$.

\section{Imaging Results}

\section{Valence cues}

Non-drug effects. A main effect of GROUP was observed across multiple MCL DA ROIs. Smokers showed decreased activation in response to both positive and negative valence cues as compared with non-smokers (Figure 1). These effects were observed in left NAc, bilateral caudate (for positive valence cues), and left caudate (for negative valence cues), right putamen, and bilateral ACC. Only the SFG ROI failed to show the GROUP effect. This widespread reduction in valence processing for smokers is consistent with previous results in an independent cohort (Rose et al, 2013).

Drug Effects: Putamen. When considering positively valenced cues, a GROUP $\times$ PILL $\times$ PATCH interaction was observed bilaterally in the tail of the putamen (Figure 2a). Consistent with previous results (Chaudhri et al, 2006,Rose et al, 2013), both smokers and non-smokers showed enhanced activation in response to nicotine administration while on the placebo pill. Interestingly, nicotine administered to abstinent smokers seemed to normalize activity to levels comparable to that of non-smokers under placebo. This effect was decreased in smokers administered VAR, but enhanced in non-smokers administered VAR. Notably, these observations did not follow the hypothesized partial agonist/ antagonist profile of VAR, as the combination of VAR and nicotine enhanced putamen activation in non-smokers. No drug effects were observed in putamen during processing of negatively valenced cues.

Drug effects: ACC. A main effect of PATCH was observed following positive valence cues such that for both smokers

\section{a}
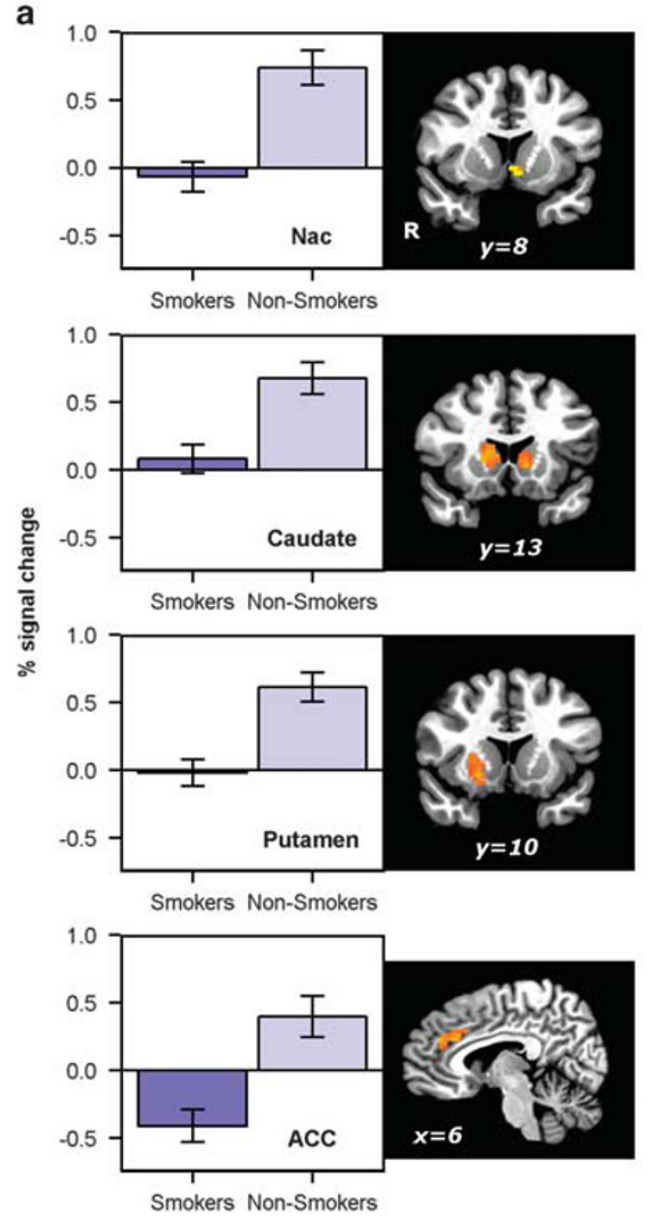

b
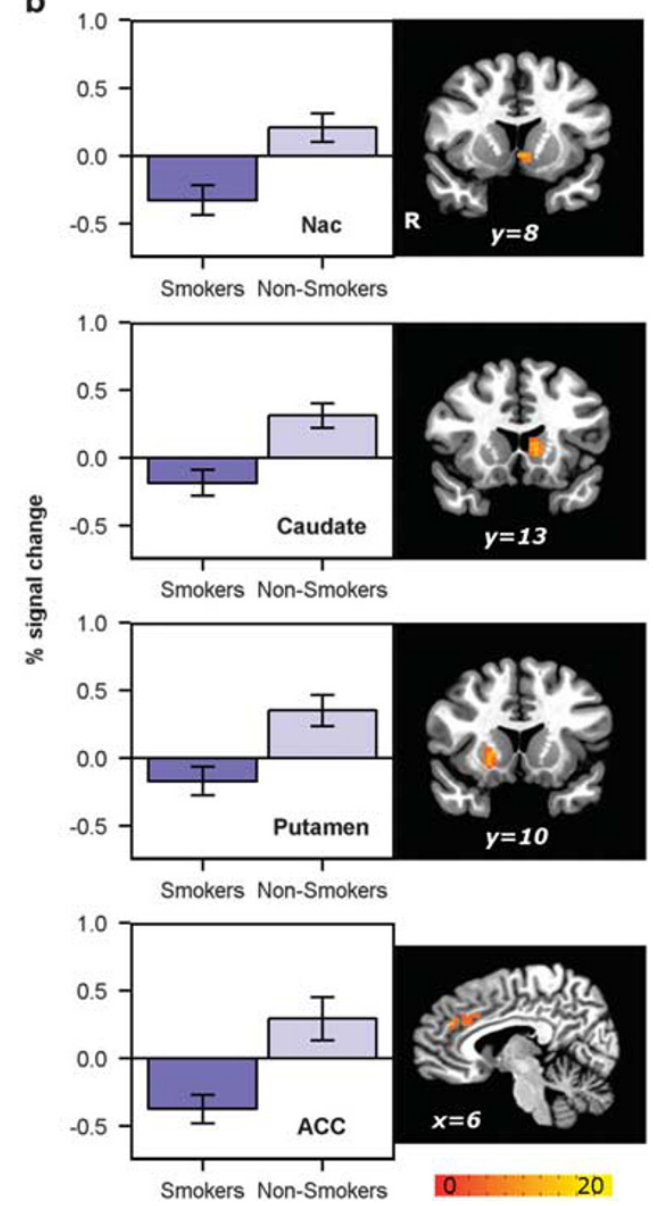

Figure I GROUP (smoker/non-smoker) effects within the mesocorticolimbic pathway (MCL) for (a) positive and (b) negative valence cues. For both positive and negative valence cues, smokers show reduced activation vs non-smokers. Error bars reflect SEM. NAc, nucleus accumbens; ACC, anterior cingulate. 

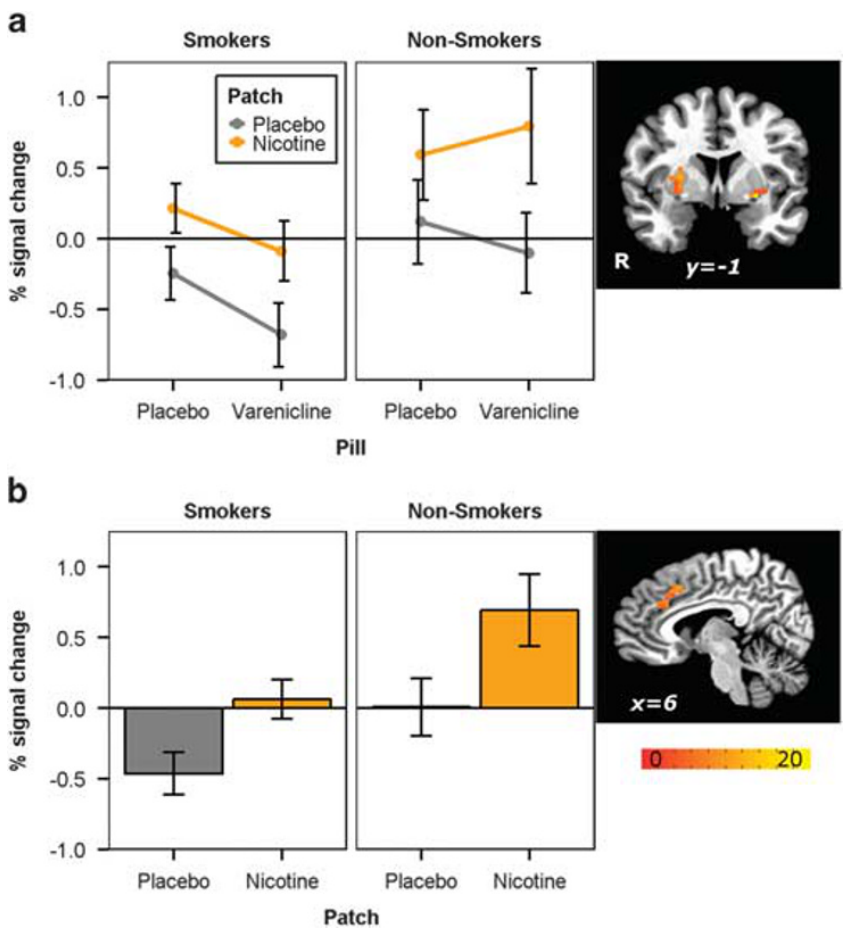

Figure 2 Drug administration effects for positive valence cues with the (a) Putamen, where a GROUP $\times$ PILL $\times$ PATCH interaction is observed and (b) anterior cingulate cortex (ACC), where a main effect of PATCH is observed. Nicotine reduces deactivation in smokers and increases activation in non-smokers. Importantly, smokers who were administered nicotine show activation similar to non-smokers who were administered placebo. Error bars reflect SEM.

and nonsmokers, nicotine ( $v s$ placebo patch) enhanced activation within ACC (Figure 2b). In contrast, no drug effects were observed in ACC during processing of negatively valenced cues.

\section{Magnitude cues}

Non-Drug Effects. A main effect of MAGNITUDE was observed bilaterally in NAc, caudate, putamen, and ACC such that higher gain magnitude cues were associated with enhanced activation and higher loss magnitude cues were associated with decreased deactivation, consistent with previous reports (Rose et al, 2013, Yacubian et al, 2006). A similar MAGNITUDE effect was observed in SFG, but only for gain magnitude cues (Figure 3a). In addition, a main effect of GROUP was observed within the bilateral caudate in response to magnitude loss cues such that smokers showed decreased deactivation when compared with non-smokers (Figure 3b).

Drug Effects: Putamen. For both gain and loss magnitude cues, main effects of PATCH were observed in the head of the putamen. For both smokers and non-smokers, nicotine decreased deactivation bilaterally during gain magnitude processing, and in the right putamen during loss magnitude processing. (Figure 4a)

When considering gain magnitude cue presentation, a GROUP $\times$ PATCH interaction was observed in the tail of the left putamen such that nicotine ( $v$ s placebo patch) enhanced deactivation in smokers, whereas decreasing deactivation in non-smokers (Supplementary Fig S5). Finally, a MAGNITUDE $\times$ PILL interaction was observed within the putamen such that VAR ( $v s$ placebo pill) was associated with enhanced deactivation following large losses (Supplementary Fig S6a).

Drug Effects: ACC. When considering gain magnitude cues, a GROUP $\times$ MAGNITUDE $\times$ PILL interaction was observed in the dorsal ACC (Figure 4b). Independent follow-up tests to characterize the observed three-way interaction showed a MAGNITUDE $\times$ PILL interaction in smokers that was absent in non-smokers. Simple main effects of PILL at either high or low magnitude gain levels were not significant. These effects are independent of the presence of nicotine.

When considering loss magnitude cues, a GROUP $\times$ PILL interaction was observed within rostral ACC. Smokers showed greater deactivation in response to VAR as compared with non-smokers. This relationship was reversed in the absence of VAR, with smokers showing decreased deactivation when compared with non-smokers (Supplementary Fig S6b).

\section{DISCUSSION}

Nicotine and VAR independently and differentially influenced anticipatory reward processing within the rewardrelated circuitry of the MCL DA pathway in acutely abstinent smokers. During gain magnitude processing, nicotine enhanced activation in the putamen-a dorsal striatal structure associated with habitual responding-whereas VAR downregulated gain magnitude processing in the ACC-a brain region linked to attentional control. These findings suggest that, in addition to its role in mitigating the withdrawal symptoms localized to the limbic circuitry (Sutherland et al, 2013a,b), VAR may aid in smoking cessation, at least in part, by reducing the salience of anticipated rewards by reducing the difference between high- and low-magnitude gain cues during the initial stages of drug administration when it is clinical practice to begin VAR administration while still smoking as usual.

We probed the neurobiology of anticipatory reward processing using a revised monetary incentive delay task in cohorts of smokers and non-smokers. We used a pharmacodynamic model-driven hypothesis to probe the putative partial agonist/antagonist profile of VAR and its ability to alter reward processing as a possible mechanism of its efficacy. Unexpectedly, and in contrast to that observed for affective cue processing (Sutherland et al, 2013a,b), no evidence of a partial agonist profile for VAR or the hypothesized nicotine $\times$ VAR interaction was observed across either positive and negative valence cue or gain and loss magnitude cue processing. Instead, VAR but not nicotine diminished the impact of reward magnitude on ACC activity, while nicotine but not VAR enhanced ACC processing when anticipating a gain $v s$ a loss.

The effects of acute nicotine within the ACC and putamen are consistent with the allostatic model of addiction (Koob and Le Moal, 2008). That is, chronic exposure to nicotine in smokers dysregulates the reward system-namely the deficit in activation observed throughout the MCL circuit in the current task. For minimally abstinent smokers, the 
a
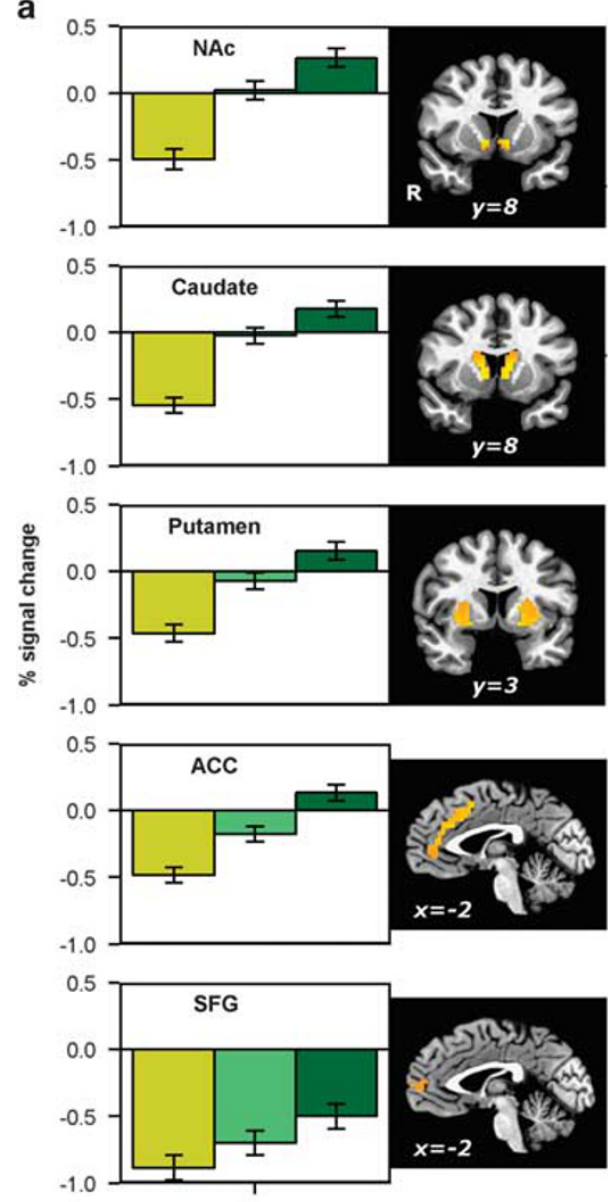

c

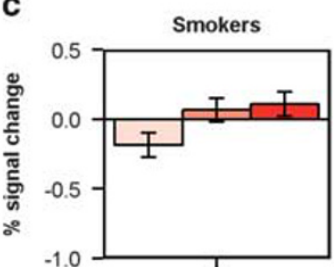

b
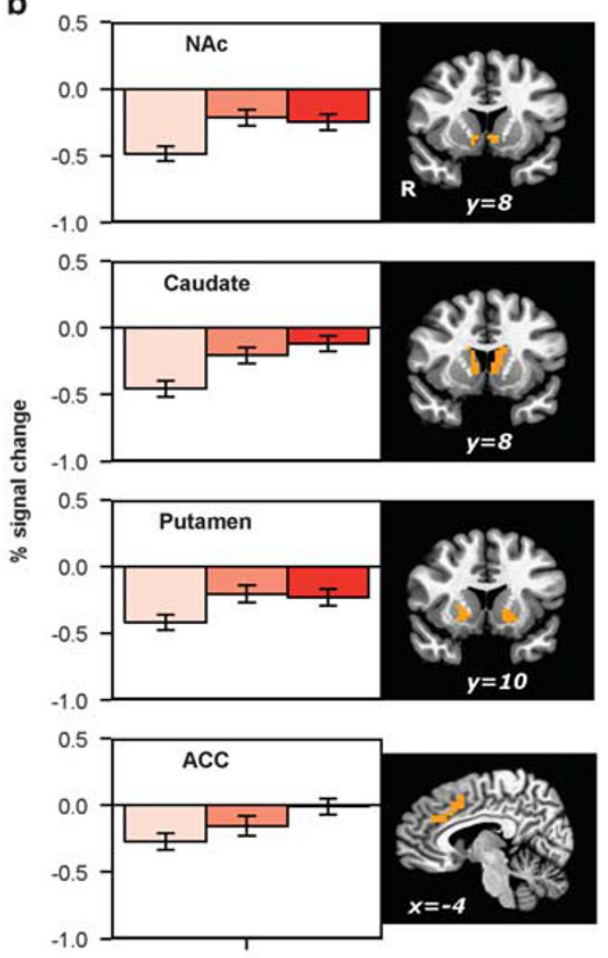

Mag Gain $\square$ Low $\square$ Med $\square$ High

Mag Loss $\square$ Low $\square_{\text {Med }}^{\text {High }}$

Figure 3 MAGNITUDE (high/medium/low) effects collapsed across both groups within the mesocorticolimbic pathway (MCL) of (a) magnitude gain and (b) magnitude loss cues showing enhanced activation for higher gain magnitude cues and decreased deactivation for higher loss magnitude cues. (c) GROUP effect within caudate showing decreased deactivation for smokers vs non-smokers. Error bars reflect SEM.

administration of nicotine assuages this deficit and returns both ACC and putamen processing to the 'normal', basal level of non-smokers. Importantly, VAR did not elicit a blunted version of these nicotine-induced enhancing effects in either the ACC or putamen. In fact, the observed GROUP $\times$ PILL $\times$ PATCH interaction in the putamen was driven by the additive enhancing effects of simultaneously administered nicotine and VAR in non-smokers as opposed to smokers.

Deficits in ACC activation have been identified as a putative source for the increased impulsivity of substance abusers (Goldstein and Volkow, 2011; Kaufman et al, 2003) and smokers specifically (Luijten et al, 2011). In addition to its role in inhibitory processing, the ACC plays a prominent role as a superordinate hub for executive control across a wide range of executive functions (Niendam et al, 2012), including monitoring for salient cues (Carter and Van Veen 2007; Ridderinkhof et al, 2004). VAR appears to reduce the salience of primary rewards and their subsequent attentional bias by attenuating ACC processing.

The ACC has been implicated in reward-mediated changes in attentional salience (Hickey and van Zoest, 2012) via an interaction with the MCL DA system (Berridge and Robinson, 1998). Enhanced ACC activation is associated with salient, attention-capturing events, even in the absence of competing stimuli. Smokers exposed to smoking-related cues showed enhanced ACC response related to increased attentional resource allocation and motor planning (Brody 


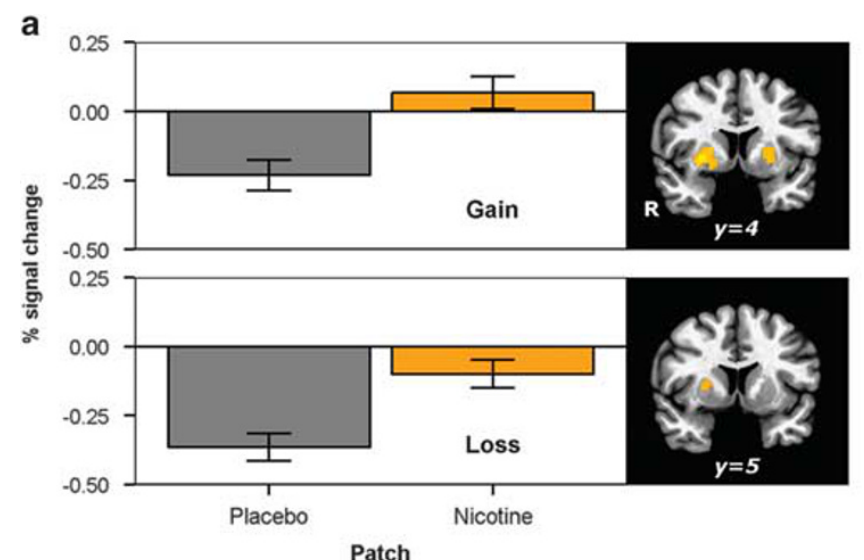

b

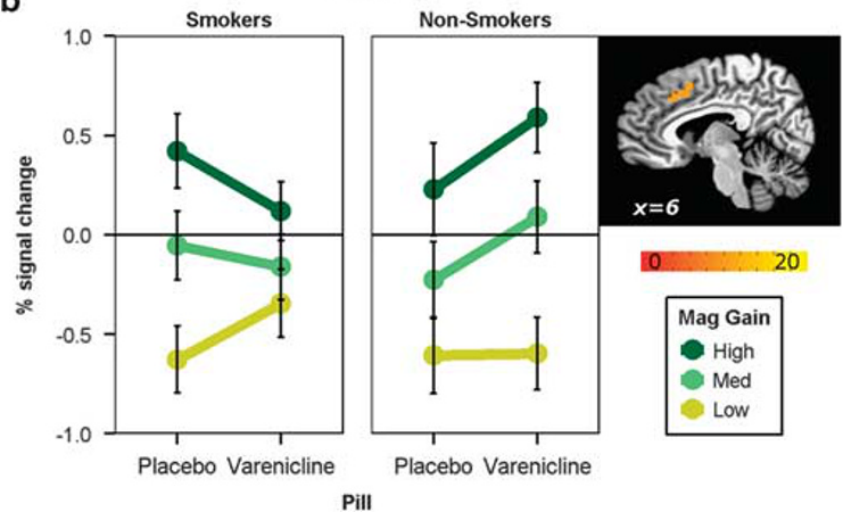

Figure 4 Drug effects on magnitude cue processing in the mesocorticolimbic pathway (MCL) for (a) gain and loss magnitude cues in the putamen. A main effect of PATCH shows nicotine decreased deactivation for both smokers and non-smokers. (b) Gain magnitude cues in the anterior cingulate cortex (ACC) where a GROUP $\times$ MAGNITUDE $\times$ PILL interaction was observed. Smokers showed decreased differentiation between gain magnitude cues when administered VAR. A MAGNITUDE $\times$ PILL interaction was observed in smokers in the absence of simple main effects of PILL at either high or low MAGNITUDE. The plot of the observed interaction is included to aid in interpretation. However, no statistical inference should be made from the plot; as such a selective analysis is likely to overstate the observed effects (that is, Kriegeskorte et al, 2009). Error bars reflect SEM.

et al, 2002; Hester and Luijten, 2014; Zhang et al, 2011), and the magnitude of ACC activation in response to smoking cues was positively correlated with self-reported cravings in abstinent smokers (McClernon et al, 2009). Moreover, the attentional biasing effects of reward are not limited to drugrelated stimuli. In a cohort of opiate-addicted participants, monetary reward was seen to bias attentional processing (Anderson et al, 2013). Together, these findings suggest that ACC governs attentional control generally under the influence of reward-related cues.

Our finding within the ACC in smokers that incentive salience, a construct strongly implicated in addiction (Berridge and Robinson, 1998), is reduced by VAR reveals a previously undescribed function of VAR unique to anticipatory reward cue processing. This informs the underlying neurobiological mechanisms of VAR's efficacy in smoking reduction. The observed downregulation of ACC is directionally consistent with VAR-mediated reductions in smoking-cue-related medial OFC activation in smokers
(Franklin et al, 2011), as well as behavioral and subjective reports of reductions in nicotine craving in smokers administered VAR (Brandon et al, 2011; Patterson et al, 2009).

These effects in smokers are driven by a MAGNITUDE $\times$ PILL interaction in the ACC. Qualitatively, the difference in ACC activation in response to large $v s$ small-magnitude gain cues is reduced in the presence of VAR. This downregulation of reward processing appears similar to the behavioral deficit in reward processing previously linked to withdrawalprecipitated relapse (Pergadia et al, 2014). However, the observed group specificity of our effect seen in smokers but not in non-smokers suggests that VAR may downregulate the processing of high value cues-for example, smokingrelated cues-while simultaneously upregulating the salience of lower-value cues (which would be therapeutically useful when one is trying to avoid smoking and enhance alternative reinforcers). Thus, we speculate that the differential effects of VAR across levels of gain magnitude may protect treatmentseeking smokers from nicotine withdrawal-precipitated relapse. Indeed, VAR downregulates the salience of smoking-related cues in both current (Brandon et al, 2011) and abstinent (Patterson et al, 2009) smokers.

The results of this study should be considered in view of a number of design limitations. The complexity of the experimental design could introduce the possibility of an increase in type I error. However, the hypotheses presented are based on prior evidence of a GROUP (smokers, non-smokers) $\times$ PILL (VAR, placebo) $\times$ PATCH (nicotine and placebo) interaction (Sutherland et al, 2013a,b) as well previously demonstrated effects of nicotine delivered alone in five MCL DA pathway ROIs while a separate cohort of smokers performed the identical MID-R task (Rose et al, 2013). Because of our strong a priori hypothesis and replication of nicotine-only findings from Rose et al (2013), this approach provides protection from type I error while simultaneously guarding against type II errors (that is, Lieberman and Cunningham, 2009). In addition, future studies should consider a wider range of pharmacological probes to characterize the mechanism responsible for the downregulation of reward processing in smokers. Finally, the current results do not address the effects of VAR during protracted abstinence, where the dynamics of reward processing may be further altered.

The dissociation between the observed effects of nicotine and VAR may inform why VAR is a successful pharmacotherapy for smoking cessation when compared with nicotine replacement therapy (NRT; Aubin et al, 2008). In the acute nicotine deprivation model employed in the current study, nicotine reduces deactivations in the MCL circuitry of smokers, 'normalizing' their reward-related processing to that seen in non-smokers. Conversely, VAR selectively downregulates the processing of gain magnitude cues in smokers, and reduces reward-related processing. Thus, while both NRT and VAR have been shown to obviate affective disruptions associated with nicotine withdrawal in a similar manner, only VAR simultaneously reduces the salience of anticipated rewards along with amelioration of negative affective components of withdrawal.

Addiction is a multidimensional neuropsychiatric disease (Goldstein and Volkow, 2002; Hyman et al, 2006; Noël et al, 2013) such that the dysregulations associated with 
compulsive drug taking manifest across multiple cognitive and affective domains. Successful treatment is likely to target multiple brain systems, networks, and circuits influenced by chronic exposure to nicotine. Our findings suggest that VAR's proven clinical efficacy is due not only to its previously described effects within the amygdala and connected limbic circuitry (Sutherland et al, 2013a,b) where it does act as a partial nicotine agonist/antagonist, but additionally to its presently described role in downregulating the magnituderelated anticipatory processing of impending rewards. Crucially, these two effects of VAR appear to rely on different mechanisms and likely act synergistically to produce its clinical efficacy.

\section{FUNDING AND DISCLOSURE}

This study was supported by the National Institute on Drug Abuse (NIDA) Intramural Research Program (JRF, MTS, BJS, TJR, and EAS). Additional funding was received from the National Institutes of Health grant K01-DA037819 (MTS). LEH receives support from the National Institute of Health grants R01-MH085646, R01-DA027680, and P50$\mathrm{MH} 103222$, and unrestricted research funding from Mitsubishi, Your Energy Systems LLC, and Pfizer. Pfizer is the manufacturer of varenicline.

\section{ACKNOWLEDGMENTS}

We thank Allison Carroll, Kim Slater, Loretta Spurgeon, Eliscia Smith, Angela Neal, and the NIDA-IRP nursing, pharmacy, and recruitment staff for their assistance with data collection.

\section{REFERENCES}

Anderson BA, Faulkner ML, Rilee JJ, Yantis S, Marvel CL (2013). Attentional bias for nondrug reward is magnified in addiction. Exp Clin Psychopharmacol 21: 499.

Aubin HJ, Bobak A, Britton JR, Oncken C, Billing CB, Gong J, Reeves KR (2008). Varenicline versus transdermal nicotine patch for smoking cessation: results from a randomized open-label trial. Thorax 63: 717-724.

Berridge KC, Robinson TE (1998). What is the role of dopamine in reward: hedonic impact, reward learning, or incentive salience? Brain Res Rev 28: 309-369.

Brandon TH, Drobes DJ, Unrod M, Heckman BW, Oliver JA, Roetzheim RC, Small BJ (2011). Varenicline effects on craving, cue reactivity, and smoking reward. Psychopharmacology 218: 391-403.

Brody AL, Mandelkern MA, London ED, Childress AR, Lee GS, Bota RG, Jarvik ME (2002). Brain metabolic changes during cigarette craving. Arch Gen Psychiatry 59: 1162-1172.

Carter CS, Van Veen V (2007). Anterior cingulate cortex and conflict detection: an update of theory and data. Cogn Affect Behav Neurosci 7: 367-379.

Chaudhri N, Caggiula AR, Donny EC, Palmatier MI, Liu X, Sved AF (2006). Complex interactions between nicotine and nonpharmacological stimuli reveal multiple roles for nicotine in reinforcement. Psychopharmacology 184: 353-366.

Chen G, Saad ZS, Britton JC, Pine DS, Cox RW (2013). Linear mixed-effects modeling approach to FMRI group analysis. Neuroimage 73: 176-190.

Coe JW, Brooks PR, Vetelino MG, Wirtz MC, Arnold EP, Huang J, O'Neill BT (2005). Varenicline: an $\alpha 4 \beta 2$ nicotinic receptor partial agonist for smoking cessation. J Med Chem 48: 3474-3477.
Cox RW (1996). AFNI: software for analysis and visualization of functional magnetic resonance neuroimages. Comput Biomed Res 29: $162-173$.

De Biasi M, Dani JA (2011). Reward, addiction, withdrawal to nicotine. Annu RevNeurosci 34: 105.

Everitt BJ, Robbins TW (2005). Neural systems of reinforcement for drug addiction: from actions to habits to compulsion. Nat Neurosci 8: 1481-1489.

Exley R, Maubourguet N, David V, Eddine R, Evrard A, Pons S, Faure P (2011). Distinct contributions of nicotinic acetylcholine receptor subunit $\alpha 4$ and subunit $\alpha 6$ to the reinforcing effects of nicotine. Proc Natl Acad Sci USA 108: 7577-7582.

Faessel HM, Gibbs MA, Clark DJ, Rohrbacher K, Stolar M, Burstein AH (2006). Multiple-dose pharmacokinetics of the selective nicotinic receptor partial agonist, varenicline, in healthy smokers. J Clin Pharmacol 46: 1439-1448.

Franklin T, Wang Z, Suh JJ, Hazan R, Cruz J, Li Y, Childress AR (2011). Effects of varenicline on smoking cue-triggered neural and craving responses. Arch Gen Psychiatry 68: 516-526.

Friedman L, Glover GH, Krenz D, Magnotta V (2006). Reducing inter-scanner variability of activation in a multicenter fMRI study: role of smoothness equalization. Neuroimage 32: 1656-1668.

Fucito LM, Toll BA, Wu R, Romano DM, Tek E, O’Malley SS (2011). A preliminary investigation of varenicline for heavy drinking smokers. Psychopharmacology 215: 655-663.

Goldstein RZ, Volkow ND (2002). Drug addiction and its underlying neurobiological basis: neuroimaging evidence for the involvement of the frontal cortex. Am J Psychiatry 159: 1642-1652.

Goldstein RZ, Volkow ND (2011). Dysfunction of the prefrontal cortex in addiction: neuroimaging findings and clinical implications. Nat Rev Neurosci 12: 652-669.

Hendrickson LM, Zhao-Shea R, Pang X, Gardner PD, Tapper AR (2010). Activation of $\alpha 4^{*}$ nAChRs is necessary and sufficient for varenicline-induced reduction of alcohol consumption. J Neurosci 30: 10169-10176.

Hester R, Luijten M (2014). Neural correlates of attentional bias in addiction. CNS Spectr 19: 231-238.

Hickey C, van Zoest W (2012). Reward creates oculomotor salience. Curr Biol 22: R219-R220.

Hyman SE, Malenka RC, Nestler EJ (2006). Neural mechanisms of addiction: the role of reward-related learning and memory. Annu Rev Neurosci 29: 565-598.

Kahneman D, Tversky A (1979). Prospect theory: an analysis of decision under risk. Econometrica 263-291.

Kaufman JN, Ross TJ, Stein EA, Garavan H (2003). Cingulate hypoactivity in cocaine users during a GO-NOGO task as revealed by event-related functional magnetic resonance imaging. J Neurosci 23: 7839-7843.

Knutson B, Westdorp A, Kaiser E, Hommer D (2000). FMRI visualization of brain activity during a monetary incentive delay task. Neuroimage 12: 20-27.

Knutson B, Adams CM, Fong GW, Hommer D (2001). Anticipation of increasing monetary reward selectively recruits nucleus accumbens. J Neurosci 21: RC159.

Koob GF, Le Moal M (2008). Neurobiological mechanisms for opponent motivational processes in addiction. Philos Trans $R$ Soc B Biol Sci 363: 3113-3123.

Koob GF, Volkow ND (2009). Neurocircuitry of addiction. Neuropsychopharmacology 35: 217-238.

Kriegeskorte N, Simmons WK, Bellgowan PS, Baker CI (2009). Circular analysis in systems neuroscience: the dangers of double dipping. Nat Neurosci 12: 535-540.

Lieberman MD, Cunningham WA (2009). Type I and Type II error concerns in fMRI research: re-balancing the scale. Soc Cogn Affect Neurosci 4: 423-428.

Luijten M, Littel M, Franken IH (2011). Deficits in inhibitory control in smokers during a Go/NoGo task: an investigation using event-related brain potentials. PLoS ONE 6: e18898. 
McClernon FJ, Kozink RV, Lutz AM, Rose JE (2009). 24-h smoking abstinence potentiates fMRI-BOLD activation to smoking cues in cerebral cortex and dorsal striatum. Psychopharmacology 204: 25-35.

McKee SA, Harrison EL, O'Malley SS, Krishnan-Sarin S, Shi J, Tetrault JM, Balchunas E (2009). Varenicline reduces alcohol selfadministration in heavy-drinking smokers. Biol Psychiatry 66: 185-190.

Niendam TA, Laird AR, Ray KL, Dean YM, Glahn DC, Carter CS (2012). Meta-analytic evidence for a superordinate cognitive control network subserving diverse executive functions. Cogn Affect Behav Neurosci 12: 241-268.

Noël X, Brevers D, Bechara A (2013). A neurocognitive approach to understanding the neurobiology of addiction. Curr Opin Neurobiol 23: 632-638.

O'Doherty JP, Deichmann R, Critchley HD, Dolan RJ (2002). Neural responses during anticipation of a primary taste reward. Neuron 33: 815-826.

Oncken C, Gonzales D, Nides M, Rennard S, Watsky E, Billing CB, Reeves K (2006). Efficacy and safety of the novel selective nicotinic acetylcholine receptor partial agonist, varenicline, for smoking cessation. Arch Int Med 166: 1571-1577.

Patterson F, Jepson C, Strasser AA, Loughead J, Perkins KA, Gur RC, Lerman C (2009). Varenicline improves mood and cognition during smoking abstinence. Biol Psychiatry 65: 144-149.

Pergadia ML, Der-Avakian A, D’Souza MS, Madden PA, Heath AC, Shiffman S, Pizzagalli DA (2014). Association between nicotine withdrawal and reward responsiveness in humans and rats. JAMA Psychiatry 71: 1238-1245.

Ridderinkhof KR, Ullsperger M, Crone EA, Nieuwenhuis S (2004). The role of the medial frontal cortex in cognitive control. Science 306: $443-447$.
Rollema H, Hajós M, Seymour PA, Kozak R, Majchrzak MJ, Guanowsky V, Williams KE (2009). Preclinical pharmacology of the $\alpha 4 \beta 2 \mathrm{nAChR}$ partial agonist varenicline related to effects on reward, mood and cognition. Biochem Pharmacol 78: 813-824.

Rose EJ, Ross TJ, Salmeron BJ, Lee M, Shakleya DM, Huestis MA, Stein EA (2013). Acute nicotine differentially impacts anticipatory valence-and magnitude-related striatal activity. Biol Psychiatry 73: 280-288.

Steensland P, Simms JA, Holgate J, Richards JK, Bartlett SE (2007). Varenicline, an alpha4beta2 nicotinic acetylcholine receptor partial agonist, selectively decreases ethanol consumption and seeking. Proc Natl Acad Sci 104: 12518-12523.

Sutherland MT, Carroll AJ, Salmeron BJ, Ross TJ, Hong LE, Stein EA (2013a). Down-regulation of amygdala and insula functional circuits by varenicline and nicotine in abstinent cigarette smokers. Biol Psychiatry 74: 538-546.

Sutherland MT, Carroll AJ, Salmeron BJ, Ross TJ, Hong LE, Stein EA (2013b). Individual differences in amygdala reactivity following nicotinic receptor stimulation in abstinent smokers. Neuroimage 66: 585-593.

Tapper AR, McKinney SL, Nashmi R, Schwarz J, Deshpande P, Labaraca C, Lester HA (2004). Nicotine activation of apha4 receptors: sufficient for reward, tolerance, and sensitization. Science 306: 1029-1032.

Yacubian J, Gläscher J, Schroeder K, Sommer T, Braus DF, Büchel C (2006). Dissociable systems for gain-and loss-related value predictions and errors of prediction in the human brain. J Neurosci 26: 9530-9537.

Zhang X, Salmeron BJ, Ross TJ, Gu H, Geng X, Yang Y, Stein EA (2011). Anatomical differences and network characteristics underlying smoking cue reactivity. Neuroimage 54: 131-141.

Supplementary Information accompanies the paper on the Neuropsychopharmacology website (http://www.nature.com/npp) 